\title{
Survey on User's Micro-Environment control using Smartphone Sensors in Android
}

\author{
P.S. Borkar ${ }^{1 *}$ K. M. Surve ${ }^{2}$, S. S. Punde ${ }^{3}$, K. S. Gorde ${ }^{4}$, S. A. Jaiswal ${ }^{5}$, S. Y. Jamaiwar ${ }^{6}$ \\ ${ }^{1}$ Dept. of CSE, Priyadarshini J. L. College of Engineering, RTMNU, India \\ ${ }^{2}$ Dept. of CSE, Priyadarshini J. L. College of Engineering, RTMNU, India \\ ${ }^{3}$ Dept. of CSE, Priyadarshini J. L. College of Engineering, RTMNU, India \\ ${ }^{4}$ Dept. of CSE, Priyadarshini J. L. College of Engineering, RTMNU, India \\ ${ }^{5}$ Dept. of CSE, Priyadarshini J. L. College of Engineering, RTMNU, India \\ ${ }^{6}$ Dept. of CSE, Priyadarshini J. L. College of Engineering, RTMNU, India \\ "Corresponding Author: pradnyabokar2@gmail.com, $\quad$ Tel: +91-7219109156
}

Available online at: www.isroset.org

Received: 27/Feb/2018, Revised: 12/Mar/2018, Accepted: 31/Mar/2018, Online: 30/Apr/ 2018

\begin{abstract}
Context-awareness is obtaining more and more necessary for a variety of mobile and pervasive applications on these days good phones. Whereas human-centric contexts (e.g., indoor/ out of doors, at home/in workplace, driving/walking) are extensively researched, few makes an attempt have studied from phones perspective (e.g., on table/sofa, in pocket/bag/hand). We have a tendency to see such immediate surroundings as micro atmosphere, typically many to a dozen of centimetres, around a phone. In this study, we have a tendency to style and implement micro-environment sensing platform that mechanically records detector hints and characterizes the micro-environment of good phones. The platform runs as a daemon method on a wise phone and provides finer-grained atmosphere data to higher layer applications via programming interfaces.
\end{abstract}

Keywords- Context-awareness, micro-environment

\section{INTRODUCTION}

In our mobile all the sensors are continuously broadcasting the data. We are going to make use of that data by reading and converting it into ASCII format. Micro environment of a smartphone can be referred to attributes from several inches around the smartphone such as light, sound, touch, etc. In this work, we created a smartphone application which records sensor hints from the smartphone's sensor array and provide feedback without requiring any human interaction. We will develop various applications using that data for security as well as for saving the battery of mobile. The sensors which we are going to use in our project are Accelerometer, Light, Pressure, Proximity etc. For example, if we keep our mobile phone in a bag or pocket, it is useless to light up the screen when a phone call is coming. In addition, if a phone is kept on a sofa rather than on a desk, it is better to turn up ring volume to avoid missing calls. We will be developing different applications using mobile sensors data. Given accurate micro-environment information, a phone can adapt its behavior automatically and properly. So, we design a micro- environment sensing platform that automatically records sensor values and the micro-environment of smart phones the system runs as service to Smartphone and provides environment information to applications via programming interfaces. There are multiple sensors embedded in mobile device. We can clear. They make the application which operates on the data which is broadcasted by a single sensor. That means this application runs continuously and uses the battery. They have not made any supportive application which saves the battery. We make many applications which come under optimization and security domain. Idea of microenvironment sensing is made on each context awareness and context sensing applications. Our project comprise of different modules like automatic call picker, PS sensor used to trace the location if operator enters the wrong pattern, for security purpose pressure sensor is used, close environment identification by using sensor for battery saving purpose etc. To making our application more efficient we focuses on battery optimization a micro- 
environment sensing platform which conducts atmospheric sensing from smart phones perspectives, which records sensor hints automatically and micro-environment of smart phones will be characterize. The platform runs in exceptional process on a good judgmental phones and provide high quality information to higher level applications using programming interfaces. On the opposite hand of sensors aims to detect immediate or fastest detection of surrounding around a phone. In Detection of Interaction is actually determines that user is currently interact with mobile devise or not. Such interaction is occur when phone is in user's hand. Recognition of Local Placements determine and define daily placements of phones such as in hand, in pocket, in bags etc. First of all by using ambient illuminative condition around the phone it is detect that phone is in hand. Micro-environment sensing provides a multidimensional, phone-oriented environment sensing service for upper layer applications and is orthogonal to the same efforts.

The organization of the paper is as follow, Section I contains the introduction of User's Micro-Environment control using smartphone sensors in Android, Section II contain the literature review for the User's MicroEnvironment control using smartphone sensors in Android, Section III contain the methodology of User's MicroEnvironment control using smartphone sensors in Android, Section IV concludes research work with future directions.

\section{LITERATURE REVIEW}

There are various microenvironment sensing with sensors approaches used in development of Android application for energy saving.

Zheng Yang [1] SHERLOCK consists of a smartphone equipped with sensors to record and infer from that data the context in which the phone is placed at any given time. In this paper a prototype was run on an android based platform and over a period of time readings were taken as input and the efficiency of the system is concluded as having high sensing accuracy, being energy efficient and providing a rapid development system. The various activities that are detected using this system include: Moving \&Walking Detection, Local Placement Recognition, Phone-in-hand Identification, On-body Placement Recognition, Phone Interaction Detection, and Backing Material Detection.
Derick A. Johnson MIROAD considers the use of smartphones to find out the driving style which can be put into two categories: Aggressive and Non-aggressive [2].

This system employs a non-invasive method with which it can record and sense the activities of user while driving to determine whether or not they are driving safely. The sensors used here are: Camera (often multiple), Microphone (often multiple), and 3-axis Accelerometer, 3axis Gyroscope, Proximity, Ambient Light, Touch, Magnetometer (compass), and GPS.

The system is named as MIROAD: A Mobile-SensorPlatform for Intelligent Recognition of Aggressive Driving. Two methods are used here: Driving Event Recognition, Gesture Recognition. When the MIROAD application is started, it can be in one of two modes: Active: data is only stored in an event when potentiallyaggressive activity is detected or passive: all the data is recorded all the time for analysis which is done at some other time than current time. Algorithms used here are detection and recognition also endpoint detection is used to detect the start and end point of manoeuvres like turning hard left or right etc.

Jilong Liao which focuses on the concept of context based input. The source of such input is through the smartphones which include the sensors as part of their internal devices which are used to read the microenvironment. This collection of data is called raw data collection which is the first step in converting such type of data into the form of sentences which are highly usable and human readable. Various machine learning algorithms are used to understand and learn the various contexts and thus infer the events accordingly. [3] The various sensors used are accelerometer and gyroscope which collect contextual data. Classification and event generation is done then the recorded data in digital diary is used to identify the patterns and behaviour of the user. This is a user-centric application which allows a person to log their daily activities which does not require their involvement. This results in a digital diary which is able to record all the data non-invasively and without any mistakes (i.e. in concise and precise manner). Two types of models are introduced here to enhance the ability of a smartphone sensor to infer and predict the activity's type efficiently. These algorithms are namely sustainable mining model and narrative structured sentence model. Steps in this methodology used: 
System Framework, Raw Data Collection, Context Analysis, Event Personalization, Diary Generation.

There are many data mining based applications in this paper which are available like health management, elder care etc. These all types of applications need to know about the user's activities that occur in his life on a daily basis. So we need a human activity recognition system to know the tasks that are performed which will result into identifying the behavioural pattern of that user. The sensors like gyroscope and accelerometers are used to record data of activities like walking and sitting. This application has - Inputs: Sensors, Outputs: Activities. The core techniques used here are: Raw data collection, Preprocessing: De-noising and segmentation, Feature computation, Classification. The challenges are: Subject sensitivity, Location sensitivity, Activity complexity, Insufficient training set, Energy and resource constrains. The applications here are: Daily life monitoring, Elderly and youth care, Personal biometric signature. [4]

Various types of sensors are present in a mobile device like camera, microphones and accelerometers which are used to perform task of video, audio and acceleration sensing. In [5] only one sensor i.e. an accelerometer is studied thoroughly and its working, implementation and usefulness is studied. The only specification for the success of this application is the presence of an accelerometer which is nowadays available in all kind of smartphone which might be an iPhone or some simple smartphone contain android as its operating system. Accelerometers are used to measure: All three dimensions, Detection of mobile phone's orientation, they can detect earth gravity. Initially this sensor was used for screen rotation and game playing but over time more of its usefulness was discovered in realizing human activity recognition. Six activities namely are: standing, ascending stairs, jogging sitting, walking and descending stairs are considered here and accelerometer is used to measure an identifying them. Different types of graphs were procured related to all these activities and then used the results in application of various functions. The various tasks are: Data Collection, Feature Generation \& Data Transformation, and The Activities.

Mostly sensor application in smartphones are used to identify simple activities such as walking sleeping etc. on the basis of locomotion of the human body, extensive inference algorithms use many different body sensors to recognize a certain human activity. [6] Stresses on finding out the complex tasks like cooking and uses only two sensors namely accelerometer and gyroscope to find out the activity instead of body sensors. Supervised learning algorithms are used to recognize the complex user activities and how they differ from simple activities. When the experiment was completed it was discovered that prediction on complex activities is very poorly done compared to simple ones. But on the other hand these algorithms were able to work on complex activities to some measure and were also able to easily identify simple tasks. There are various activities namely: Data Collection, Activities, Feature Extraction, and Classification.

Recommendation system is one of the important methods of information retrieval. Environmental sensors are used to gather user's data from their daily activities. Earlier social networks used social graphs to find friends but this recommender system uses mobile sensor data to infer the life style and habit to recommend them to other people with similar choices. [7] Text and data mining activities are performed on raw data collected from user activities which is called here as life documents. Algorithm named is a data mining and extraction tool used in this system. The process in this system helps find people friends who cannot be easily searched by basic search methods and queries. Friend matching graphs are used to match and evaluate the likeness and feedback methods help increase the recommendation accuracy.

The author has proposed PerFallD, utilizing mobile phones as a platform for pervasive fall detection system whose only requirement is a mobile Environmental Monitoring phone which has an accelerometer. In [8] there is Acceleration-based. Detection approach algorithm is used. PerFallD has few false positives and false negatives; it is available in both indoor and outdoor environment; it is user-friendly, and it does not require extra hardware and service cost. It is also lightweight and power efficient. The design of this system is general and it is not constrained to a particular brand or type of mobile phone. It is divided into five major components: user interface, monitoring daemon, data processing, alert notification and system configuration.

Nericell is a system that performs rich sensing by piggybacking on smart phones that users carry with them in normal course. The authors have focused specifically on the sensing component, which uses the accelerometer, microphone, GSM radio, and/or GPS sensors in these 
phones to detect potholes, bumps, braking, and honking. In this paper there is Strongest signal (SS)-based localization algorithm is used. Nericell addresses several challenges including virtually reorienting the accelerometer on a phone that is at an arbitrary orientation, and performing honk detection and localization in an energy efficient manner. [9]

V-Track Road Traffic Delay Estimation et al have proposed a system called V-Track for travel time estimation using this sensor data and it addresses two key challenges: energy consumption and sensor unreliability. While GPS provides highly accurate location estimates, it has several limitations and considering those limitations the authors have proposed that V-Track can use alternative, less energy-hungry but noisier sensors like WiFi to estimate both a users' trajectory and travel time along the route. V-Track processes streams of time stamped, inaccurate position samples at time-varying periodicity from mobile phones. It uses a Hidden Markov Model (HMM) to model a vehicle trajectory over a block level map of the area.

The location and context switching, especially the indoor/outdoor switching, provides essential and primitive information for upper layer mobile applications. In this, author presented IO Detector: a lightweight sensing service which runs on the mobile phone and detects the indoor/outdoor environment in a fast, accurate, and efficient manner. In this, dynamic programming is used. Viterbi algorithms used for finding the most likely sequence of hidden states called the Viterbi path. Constrained by the energy budget, IO Detector leverages primarily lightweight sensing resources including light sensors, magnetism sensors, cell tower signals, etc.

APT Accurate Outdoor Pedestrian Tracking is a localization system for outdoor pedestrians with smartphones. APT performs better than the built-in GPS module of the smartphone in terms of accuracy. This is achieved by introducing a robust dead reckoning algorithm and an error-tolerant algorithm for map matching. When the user is walking with the smartphone, the dead reckoning algorithm monitors steps and walking direction in real time. It then reports new steps and turns to the mapmatching algorithm.

Skubic. M [10] designed to investigate embedded health assessment. Described a prospective study using 1-D health alerts. Clinical ratings on the health alerts were provided by clinicians and used to train and test multi-D classifiers. Author proposed a model for detecting health decline with in-home sensors. A randomized control study using this model with the hydraulic bed sensor, motion sensors, and in-home gait is underway to further test the potential of embedded health assessment. Offers the potential for improved health outcomes, reduced healthcare costs, continued independence, and better quality of life.

\section{MeTHODOLOGY}

Our application automatically records sensor data and provide input data to smartphones. The application runs as a background process on a smartphone and provides user with a micro environment using the said sensors.

Following is the proposed methodology for recognizing the task and activities sensors have to analyses the various events and the corresponding changes in them that occur over intervals of time.

STEP 1: Configuring the settings of various applications according to which threshold of different sensors can be set if required.

STEP 2: Initiating the application by turning them on such that they run continuously in the background of system and collect data as input.

STEP 3: Sensors are used to collect user contextual data which is in physical form initially and then converted into parameters that can be manipulated for deduction of events.

STEP 4: By analyzing the collected data, events are recognized and these events help in defining the activity.

STEP 5: After inferring a task an application assists user to efficiently perform activities.

STEP 6: The performance of the system is increased by a evaluating and eliminating unnecessary tasks.

STEP 7: When a certain activity occurs it is recognized by the system and accordingly the action is taken.

\section{CONCLUSION}

We will be developing an app "SmartSense" which will be a simple yet practical platform for micro-environment sensing for smartphones via collaboration among built-in sensors. The platform automatically collects sensor hints and characterizes the immediate surroundings of smartphones at centimetre level accuracy, providing finegrained environment information to upper layer applications. We shall conduct comprehensive experiments 
to evaluate this system through a prototype implementation on Android platform.

\section{REFERENCES}

[1] Z. Yang, L. Shangguan, W. Gu, Z. Zhou, C. Wu and Y. Liu, "Sherlock: Micro-environment Sensing for Smartphones", IEEE Transactions on Parallel and Distributed Systems, Vol 2. Issue 3, 2013

[2] Derick A. Johnson and Mohan M. Trivedi, "Driving Style Recognition Using a Smartphone as a Sensor Platform", Laboratory for Intelligent and Safe Automobiles (LISA) University of California, San Diego La Jolla

[3] Intelligent Transportation Systems Washington, DC, USA. Pp.1609-1615, 2011

[4] Jilong Liao, Zhibo Wang, Lipeng Wan, Qing Cao and Hairong Qi, "Smart Diary: A Smartphone-based Framework for Sensing, Inferring and Logging Users' Daily Life", pp.4-12, 2013

[5] Jennifer R. Kwapisz, Gary M. Weiss, Samuel A. Moore, “Activity Recognition using Cell Phone Accelerometers", IEEE 2014

[6] Activity recognition with smartphone sensors by Stefan Dernbach. Pp. 121-133

[7] Zhibo Wang, Jilong Liao, Qing Cao, Hairong Qi and Zhi Wang, "Friendbook: A Semantic-based Friend Recommendation System for Social Networks", IEEE, 2013

[8] J. Dai, X. Bai, Z. Yang, Z. Shen, D. Xuan. "PerFallD: A Pervasive Fall Detection System Using Mobile Phones." In PervasiveHealth'10, 2010.

[9] Prashanth Mohan, Venkata N. Padmanabhan, Ramchandran Ramjee. "Nericell: Rich Monitoring of Road and Traffic Conditionsusing Mobile Smartphones" In Mobisys 2010

[10] Marjorie Skubic 1, (Senior Member, IEEE), Rainer Dane Guevara 1, 2, and Marilyn Rantz 3, (Member, IEEE). Automated Health Alerts Using In-Home Sensors.

\section{Authors' Profile}

Mrs. Pradnya Borkar is currently working as an Assistant Professor in Priyadarshini J .L. College of Engineering, Nagpur. She has submitted Ph.D. (Computer Science \& Engg) to Rashtrasant Tukadoji Maharaj Nagpur University, Nagpur. Her area of specialization is High

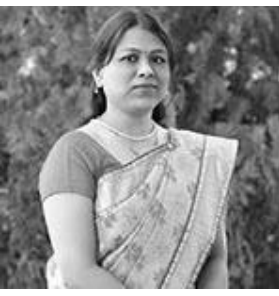
Performance Computing, Parallelization, Bioinformatics, and Database Management System. She has published research papers in renowned international journals, International and National Conference Proceedings. I have organized and attended many STTPs/Workshops/Seminars. She is a member of various professional bodies and also reviewer of international journals. She is the author of Book "Data Warehousing and Mining".

Kashish Surve is pursuing his bachelor of engineering in Computer Science \& Engineering from Priyadarshini J.L of Engineering, Rashtrasant Tukadoji Maharaj Nagpur University, Nagpur-440009, India.

Kalika Gorde is pursuing her bachelor of engineering in Computer Science \& Engineering from Priyadarshini J.L of Engineering, Rashtrasant Tukadoji Maharaj Nagpur University, Nagpur-440009, India.

Sanvid Punde is pursuing his bachelor of engineering in Computer Science \& Engineering from Priyadarshini J.L of Engineering, Rashtrasant Tukadoji Maharaj Nagpur University, Nagpur-440009, India.

Sachin Jaiswal is pursuing his bachelor of engineering in Computer Science \& Engineering from Priyadarshini J.L of Engineering, Rashtrasant Tukadoji Maharaj Nagpur University, Nagpur-440009, India.
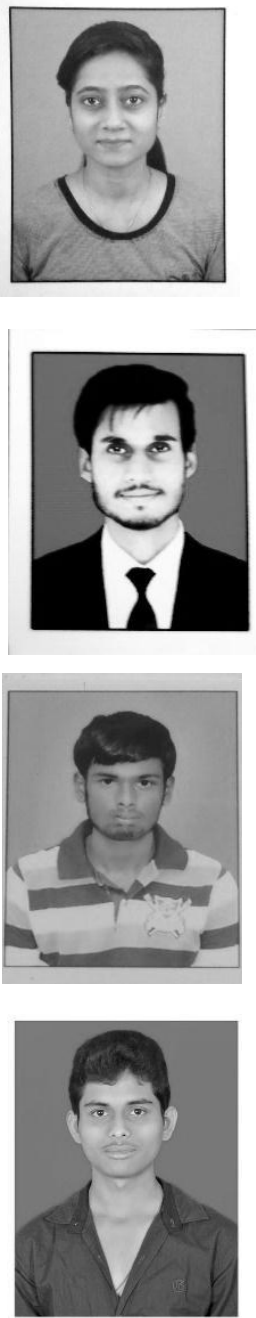

Shivam Jamaiwar is pursuing his bachelor of engineering in Computer Science \& Engineering from Priyadarshini J.L of Engineering, Rashtrasant Tukadoji Maharaj Nagpur University, Nagpur-440009, Indi

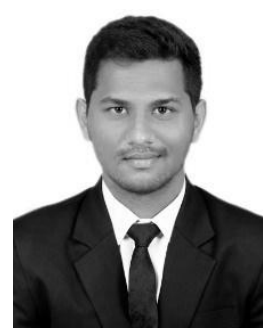

